\section{Highlights of the San Antonio Breast Cancer Symposium 2016}

\author{
John R Benson ${ }^{* 1} \&$ Ismail Jatoi ${ }^{2}$
}

The 39th annual San Antonio Breast Cancer Symposium, San Antonio, Texas, 9-13 December 2016

The 39th annual San Antonio Breast Cancer Symposium (SABCS) was convened in San Antonio, Texas, on 9-13 December 2016. More than 7000 clinicians and scientists from around the world participated in the symposium which featured a range of presentations and keynote talks pertaining to breast cancer screening, prevention, locoregional and systemic therapies. This two-part report highlights a selection of important studies presented at this premier breast cancer event with Part 1 focusing onmetastatic breast cancer, extended endocrine therapy and the prognostic significance of $B R C A 1 / 2$ gene mutations. The second part of this report will discuss a range of topics including anti-HER2 directed treatments, the impact of radiotherapy on implant and autologous flap based reconstruction, biological risk predictors for ductal carcinoma-in situ (DCIS), longer term effects of dietary fat modification and the influence of aromatase inhibitors on endothelial cell function.

First draft submitted: 27 February 2017; Accepted for publication: 20 April 2017; Published online: 26 June 2017

\section{Part 1}

In the first plenary lecture, Stephen Johnston (Royal Marsden Hospital, London, UK) discussed the management of estrogen receptor (ER)-positive metastatic breast cancer (MBC). Emerging data suggest that aromatase inhibitors (AI) alone can be improved upon as standard of care for hormonesensitive $\mathrm{MBC}$ [1]. Combination of endocrine therapy with agents targeting growth factor/cell cycle pathways can potentially delay onset of endocrine resistance. The growth of ER-positive breast cancer is dependent on cyclin D1, an ER transcriptional target. Cyclin D1 activates CDK 4/6 and this in turn controls G1-S phase transition and entry into the cell cycle. Johnston aptly commented how trials investigating CDK 4/6 inhibitors have shown 'breathtaking' results and been a 'real game changer' in first-line management of hormone receptor-positive MBC. Both the PALOMA-2 (NCT 01740427) [1] and MONALEESA-2 (NCT 01958021) [2] are Phase III studies evaluating the combination of a CDK 4/6 inhibitor with an AI using progression-free survival (PFS) as the primary end point. In the PALOMA-2 trial, patients were randomized to a combination of letrozole and palbociclib versus letrozole alone with a dramatic improvement in PFS for the combined therapy (hazard ratio [HR]: 0.58; $\mathrm{p}=0.001$ ). Likewise, the MONALESSA-2 trial revealed a significant improvement in PFS of about $40 \%$ for a combination of a CDK $4 / 6$ inhibitor (ribociclib) and letrozole (HR: 0.50). In particular, early separation of the survival curves was evident suggesting that all patients benefit from these therapies and now just a few - CDK 4/6 inhibitors possess

'Cambridge Breast Unit, Addenbrookes Hospital \& University of Cambridge, Faculty of Medical Sciences, Anglia Ruskin University, Cambridge, UK

2Division of Surgical Oncology, Dale H Dorn Chair in Surgery, University of Texas Health Science Centre, TX, USA *Author for correspondence: Tel.: +44 1223 586577; Fax:+44 1223 586932; john.benson@addenbrookes.nhs.uk

\section{KEYWORDS}

- breast conserving surgery

- extended endocrine

- mastectomy

- reconstruction $\bullet$ radiation

treatment $\bullet$ San Antonio
Future
Medicine $\mathrm{fs}$ par of 
a 'ubiquitous' target. An important aspect of combination therapies is toxicity, and CDK 4/6 inhibitors are generally well tolerated with few side effects. Therefore, the combination of an AI with a CDK 4/6 inhibitor should be considered as the new first-line line standard of care for all ER-positive MBC patients.

Second-line treatment approaches have explored the role of mTOR inhibitors and $\mathrm{PI} 3 \mathrm{~K}$ inhibitors in combination with endocrine agents [3]. Biomarkers of response are urgently needed for these newer combination endocrine therapies, which are likely to involve changes in key signaling pathways - PI3KCa, FGFR, ER/ $\mathrm{PgR}$ expression and other molecular profiles. It remains unclear whether PI3KCa is a predictor of response to mTOR inhibition with provisional data suggesting that wild-type and mutant forms of PI3KCa display similar responses. There are currently no biomarkers to predict response to CDK 4/6 inhibitors and guide clinical management on combination endocrine therapies. The affordability and availability of drugs are important issues in addition to cost and optimum dosage/duration of usage. Endocrine approaches can be used to defer the need for chemotherapy with maximum PFS of 14-16 months for single agents (AI or fulvestrant). Combination therapy with fulvestrant and CDK 4/6 inhibitors can increase median PFS to $>23$ months for firstline treatment and 10-11 months for second-line treatment.

Several trials evaluating extended adjuvant endocrine therapy were presented at SABCS2016. The Phase III DATA study randomized 1912 postmenopausal women with ER/PgR-positive breast cancer and no signs of disease recurrence to either 3 or 6 years of anastrozole therapy after $2-3$ years of adjuvant tamoxifen. Intention-to-treat analysis was conducted on 823 and 827 patients in the 3- and 6-year groups respectively and the results were presented by Vivianne Tjan-Heijnen (Maastricht University, The Netherlands). For the primary outcome measure of adapted disease-free survival (aDFS), any event (starting 3 years from randomization) was defined as invasive or noninvasive cancer recurrence, second nonbreast primary cancers and death from any cause. The secondary end point for this study was overall survival. The aDFS at 5 years was $83.1 \%$ for the 6 years group and $79.4 \%$ for the 3 years group with no statistically significant differences (HR: 0.79 [0.62-1.02]; $\mathrm{p}=0.07$ ). Despite no benefit from extended endocrine therapy for the cohort as a whole, subgroup analysis revealed a significant improvement in aDFS for patients with larger node-positive tumors that were ER/ PgR positive, HER2 negative and in receipt of previous chemotherapy (86.0 vs $75.9 \%$; HR: 0.58 [0.39-0.89]; $\mathrm{p}=0.01)$. There was no difference in adapted overall survival (HR: 0.91 [0.65-1.29]; $\mathrm{p}=0.6)$ and more prolonged anastrozole therapy was associated with increased morbidity including arthralgia, muscle pain, osteoporotic fracture and arterial thrombotic events. The authors concluded there was no benefit from extending AI therapy beyond 5 years of sequential therapy, but a subgroup of patients may derive a survival advantage. Genomic classifiers are needed to determine risk of late relapse and benefit from extended endocrine therapy.

Erik Blok (Leiden University Medical Centre, The Netherlands) followed on with presentation of results from the IDEAL trial, which randomized 1824 patients to either 2.5 or 5 years of letrozole after an initial 5 -year treatment period with tamoxifen and/or AI. Primary end point was DFS with overall survival as the secondary end point. At a median follow-up of 6.5 years, there was no statistically significant difference between the group receiving 2.5 versus 5 years of endocrine therapy (HR: 0.96 [0.76-1.20]; $\mathrm{p}=$ $0.70)$. Neither were there differences in adjusted overall survival (HR: 1.08 [0.81-1.45]; $\mathrm{p}=0.59$ ) nor for the distant metastasis-free interval. Incidence of toxicity (all grades for arthralgia, myalgia, bone fracture, osteoporosis/penia) in each group was $70 \%$ with a low number of additional adverse events attributable to extended therapy. The authors concluded that there was no benefit in extending AI therapy beyond 2.5 years for the majority of patients (i.e., 7.5 years in total of sequential therapy with tamoxifen/AI is sufficient and should not be extended out to 10 years with further AI therapy).

Elefterios Mamounas (University of Florida Health Cancer Centre, FL, USA) discussed results of the NRG Oncology/NSABP B-42 trial (NCT 00382070) that randomized 3966 patients to either 5 years of letrozole or placebo. All patients were postmenopausal and disease free after 5 years of endocrine therapy consisting of either 5 years of an AI or tamoxifen with switch to an AI after 2-3 years. There was a $15 \%$ reduction in the primary end point of DFS favoring letrozole (HR: 0.85; $\mathrm{p}=0.048$ ). This was not deemed statistically significant as 
a predefined p-value of 0.0418 was used for primary end point analysis (to account for alpha spending during preplanned interim analyses). Although no survival differences were found (HR: 1.15 [0.92-1.14]; $\mathrm{p}=0.22$ ), there was a statistically significant improvement in breast cancer free interval (HR: 0.71; $\mathrm{p}=0.003$ ) and also the cumulative incidence of distant recurrence (HR: $0.72 ; p=0.03$ ) in the letrozole arm.

As discussant for these papers, Michael Gnant (Medical University of Vienna, Austria) reminded the audience that there is a significant risk of relapse for hormone receptor-positive patients and half of these cases occur beyond the first 5 years. This justifies exploration of extended endocrine therapy as a clinical strategy [4-6]. An outstanding question is the use of extended AI therapy when these agents have been prescribed in the initial 5 years. There are important issues relating to side effects and adherence which are interrelated; in all trials of extended endocrine therapy there is a constant decline in treatment adherence and only $60 \%$ of patients in B-42 completed letrozole treatment. Symptoms of arthralgia and myalgia are particularly prominent among patients receiving prolonged AI therapy and advancing age is associated with greater risk of bone fractures. All three trials have shown negative results for the primary end point of DFS and decisions for extended endocrine therapy must balance costs and side effects. For patients who have received tamoxifen for either $2-3$ or 5 years, it is reasonable to consider extending therapy with an AI for a period of 2.5-5 years. However, for those patients who have previously been treated with an AI only, clinical caution is warranted; if patients tolerated these agents well in the first 5 years, are relatively young with good bone health, then extended endocrine therapy with an AI can be considered. Those patients deemed to be at higher risk for late relapse based on clinical-pathological factors should be considered for extended therapy and genomic tests might aid selection of these patients in the future. It should be noted that extended endocrine therapy is associated with a significant reduction in second primary breast cancers.

There is currently much interest in tumorinfiltrating lymphocytes (TiLs) in early stage breast cancer where associations with improved pCR rates, event-free, disease-free and overall survival have been reported for HER2-positive disease in retrospective analyses [7]. Stephen
Luen (Peter MacCallum Cancer Centre in Melbourne, Australia) presented results from a subanalysis (CLEOPATRA study [NCT 00567190]) of TiLs in HER2-positive advanced breast cancer where any prognostic associations are unknown. There was an $11 \%$ reduction in risk of death per $10 \%$ incremental rise in TiLs (overall survival HR: 0.89 [0.83-0.96]; $\mathrm{p}=$ $0.001)$. Moreover, overall survival was related to mean TiLs level within each treatment arm (pertuzumab vs control). Comparison of survival within each arm for $\leq 20 \%$ versus $>20 \%$ TiL levels revealed significantly higher overall survival for higher TiL levels on pooled log-rank analysis $(p<0.001)$. There was no significant interaction between TiLs effect and pertuzumab treatment and no predictive effect of TiLs with respect to pertuzumab treatment. A plot of logrelative HR for death versus stromal TiLs per $10 \%$ increment showed existence of a linear relationship between TiL effect and survival. These results suggest a favorable influence of pre-existing TiLs in the advanced setting where strategies to enhance antitumor immunity may be appropriate. Continuing on this theme Carsten Denkert (Germany) discussed the role of TiLs as a predictive and prognostic biomarker for certain immunogenic subtypes of breast cancer. He concluded that TiLs are a strong predictive marker for pathological complete response (pCR) in all tumor subtypes and pCR translates into a survival benefit for triple negative (TNBC) and HER2-positive breast cancers. No survival benefit is evident for luminal tumors although higher levels of TiLs are linked to pCR for this subtype and patients with a $\mathrm{PCR}$ have a good prognosis. Furthermore, patients without a pCR and low levels of TiLs will have a better prognosis compared with patients with higher levels of TiLs.

Results from the prospective study of outcomes in sporadic and hereditary breast cancer (POSH) study were presented by Diana Eccles (University of Southampton, UK). This study examined whether $B R C A$ gene mutations have an independent prognostic effect for patients with early stage breast cancer and known BRCA status. The primary outcome measure for this study was overall survival for breast cancer patients with and without $B R C A$ gene mutations. A total of $379 / 2759$ patients (14\%) were found to have mutations in BRCA-1 (212), BRCA-2 (170) or both. Patients with BRCA-1 mutations were younger with smaller tumor size 
and TNBC was more likely to carry a $B R C A-1$ mutation (19\%). Overall survival was found to be the same for all BRCA-positive patients versus non-BRCA-positive patients at a median followup of 8.2 years. These findings applied to both univariate (HR: 0.99 [0.80-1.23]; $\mathrm{p}=0.938$ ) and multivariate analysis (HR: 0.96 [0.77-1.21]; $\mathrm{p}=0.742$ ) and for separate analysis of $B R C A-1$ mutation carriers compared with noncarriers. Although no statistically significant difference in overall survival was found between $B R C A$ gene mutation carriers and noncarriers among all young breast cancer patients, there was a consistent $11 \%$ survival benefit at 10 years for BRCA gene mutation carriers presenting with TNBC (76.37 vs $65.85 \%$ ).
Results of the GANEA 2 trial (NCT 01221688) were presented by Jean-Marc Classe (Nantes, France). This prospective multi-institutional trial examined the performance of sentinel lymph node biopsy (SLNB) following chemotherapy depending on axillary nodal involvement at presentation. It was concluded that SLNB following neoadjuvant chemotherapy is safe when axillary nodes are not initially involved. By contrast, when there is biopsy-proven axillary node metastases prechemotherapy, SLNB alone following chemotherapy has not yet been proven to be safe [8]. There remains a clinical risk of false-negative results ( $>20 \%$ ) and this is not readily controllable. Moreover, long-term data on axillary recurrence is lacking and SLNB in

\section{EXECUTIVE SUMMARY}

- A combination of endocrine therapy with agents targeting growth factor/cell cycle pathways can potentially delay onset of endocrine resistance in metastatic breast cancer.

- An aromatase inhibitor (Al) with a CDK4/6 inhibitor should be considered as the new first-line standard of care for all estrogen receptor positive metastatic breast cancer patients.

- A combination of fulvestrant with CDK 4/6 inhibitors can increase median progression-free survival to almost 24 months for first-line treatment and almost 12 months for second-line treatment.

- There is a significant risk of relapse for hormone receptor-positive early breast cancer patients and half of this occurs beyond 5 years with a continued risk out to 20 years.

- For most patients, there is no demonstrable benefit from extending Al therapy beyond 5 years of therapy when anastrozole is sequenced after 2-3 years of tamoxifen.

- Likewise, there is no benefit from extending sequential treatment with tamoxifen and letrozole from 7.5 years out to 10 years total duration of therapy.

- There is a nonsignificant $15 \%$ reduction in disease-free survival for an additional 5 years of letrozole following 5 years of an Al or tamoxifen/Al switch.

- For those patients in receipt of tamoxifen for 2-3 or 5 years, it is reasonable to consider extending endocrine therapy with either an Al for 2.5-5 years.

- For patients previously treated with an Al only, extended endocrine therapy with an Al can be considered if tolerant of these agents and relatively young with good bone health.

- Patients at higher risk for late relapse should be recommended extended endocrine therapy.

- Pre-existing tumor-infiltrating lymphocytes have a favorable influence in the advanced setting where enhancement of antitumor immunity may be appropriate.

- Tumor-infiltrating lymphocytes are a strong predictive marker for pathological complete response for all subtypes, and this translates into a survival benefit for triple negative and HER2-positive breast cancers.

- There is no statistically significant difference in overall survival between early breast cancer patients who are known $B R C A$ gene mutation carriers and noncarriers but evidence improved survival for triple negative breast cancers among $B R C A$ gene mutation carriers.

- Rates of re-excision after breast conserving surgery have decreased consequent to a consensus statement on definition of a negative surgical resection margin was published in 2014.

- There has been an increase of $13 \%$ in breast conserving surgery and a corresponding decrease in both unilateral and bilateral mastectomy. 
the latter context therefore remains controversial. Although Classe maintained that SLNB alone in the setting of biopsy-proven node positivity prechemotherapy should not be undertaken outside clinical trials, many surgeons are adopting this approach when there is normalization of nodes after neoadjuvant chemotherapy and sentinel nodes with micrometastases are considered positive [9].

The Brinker Award lecture for scientific distinction in clinical research was delivered by Monica Morrow from Memorial SloanKettering Cancer Centre, New York and entitled "Changing paradigms in the local therapy of breast cancer: Making less more". Morrow believes that several factors have come together in the current era to create a 'perfect storm'. On one hand, there is reduced tumor burden at presentation, better systemic treatments and an absolute reduction in rates of locoregional recurrence. On the other hand, indications for systemic treatments have increased, patients are demanding 'big surgery', duration of therapies has increased and indications/fields for radiotherapy have expanded. Therefore, the incremental addition of therapies has effectively increased aggregate treatments for patients. Morrow advocates leverage of systemic treatment effects to reduce the extent of locoregional therapies; surgical paradigms for managing the breast and axillary nodes were developed circa 1970-1990 and based on the principle of tumor extirpation. There has been a trend toward polarization of breast surgery with some patients opting for unilateral breast conserving surgery (BCS) while others demand bilateral mastectomy (+/- reconstruction) for smaller unilateral cancers. Surgical re-excision for negative margins has decreased since a consensus statement on definition of a negative margin [10]; for margins of $\leq 1 \mathrm{~mm}$ re-excision rates have decreased from 43 to $30 \%$. Morrow concluded that there has been a $16 \%$ reduction in additional surgery after initial lumpectomy as a consequence of fewer patients with 'negative' margins having re-excision. There has been an increase in BCS of $13 \%$ and a corresponding decrease in both unilateral and bilateral mastectomy and thus development of evidence-based guidelines on margins has led to reduction in use of postlumpectomy surgery.

\section{Financial \& competing interests disclosure}

The authors have no relevant affiliations or financial involvement with any organization or entity with a financial interest in or financial conflict with the subject matter or materials discussed in the manuscript. This includes employment, consultancies, honoraria, stock ownership or options, expert testimony, grants or patents received or pending, or royalties.

No writing assistance was utilized in the production of this manuscript.

\section{References}

1 Finn RS. PALOMA-2: primary results from a Phase III trial of pablociclib with letrozole compared with letrozole alone in postmenopausal women with ER positive, HER2 negative advanced breast cancer. J. Clin. Oncol. 34, Abstract 507 (2016).

2 Hortobagyi GN, Stemmer S, Burris HA et al. Ribociclib as first line therapy for HRreceptor positive advanced breast cancer. N. Engl. J. Med. 375, 1738-1748 (2016).

3 Baselga J, Campone M, Piccart M et al. Everolimus in post-menopausal hormonereceptor positive advanced breast cancer. N. Engl. J. Med. 366, 520-529 (2012).

4 Goss P, Ingle J, Pritchard K et al. Extending aromatase inhibitor adjuvant therapy to 10 years. N. Engl. J. Med. 375, 209-219 (2016).
5 Davies C, Hongchao P, Godwin J et al. Long term effects of continuing adjuvant tamoxifen to 10 years versus stopping at 5 years after diagnosis of oestrogen receptor positive breast cancer: ATLAS - a randomized trial. Lancet 381(9869), 805-816 (2013).

Gray R, Rea D, Handley K et al. aTTom: long term effects of continuing adjuvant tamoxifen to 10 years versus stopping at 5 years in 6953 women with early breast cancer. J. Clin. Oncol. 31(Suppl. 1), Abstract 5 (2013).

7 Loi S, Sirtaine N, Piette F et al. Prognostic and predictive value of tumor-infiltrating lymphocytes in a Phase III randomized adjuvant breast cancer trial in node-positive breast cancer comparing the addition of docetaxel to doxorubicin with doxorubicinbased chemotherapy: BIG 02-98. J. Clin. Oncol. 31(7), 860-867 (2013).
8 Benson JR, Jatoi I. Sentinel lymph node biopsy and neoadjuvant chemotherapy in breast cancer patients. Fut. Oncol. 10(4), 577-586 (2014).

9 Boileau JF, Poirer B, Basik M et al. Sentinel node biopsy after neoadjuvant chemotherapy in biopsy-proven node-positive breast cancer: the SN FNAC study. J. Clin. Oncol. 33, 258-264 (2015).

10 Moran MS, Schnitt S, Giuliano AE et al. Society of Surgical Oncology - American Society for Radiation Oncology consensus guideline on margins for breast conserving surgery with whole breast irradiation in stage I and II invasive breast cancer. Ann. Surg. Oncol. 3, 704-716 (2014). 